\title{
Inside-Outside Estimation of a Lexicalized PCFG for German
}

\author{
Franz Beil, Glenn Carroll, Detlef Prescher, Stefan Riezler and Mats Rooth \\ Institut für Maschinelle Sprachverarbeitung, University of Stuttgart
}

\begin{abstract}
The paper describes an extensive experiment in inside-outside estimation of a lexicalized probabilistic context free grammar for German verbfinal clauses. Grammar and formalism features which make the experiment feasible are described. Successive models are evaluated on precision and recall of phrase markup.
\end{abstract}

\section{Introduction}

Charniak (1995) and Carroll and Rooth (1998) present head-lexicalized probabilistic context free grammar formalisms, and show that they can effectively be applied in inside-outside estimation of syntactic language models for English, the parameterization of which encodes lexicalized rule probabilities and syntactically conditioned word-word bigram collocates. The present paper describes an experiment where a slightly modified version of Carroll and Rooth's model was applied in a systematic experiment on German, which is a language with rich inflectional morphology and free word order (or rather, compared to English, free-er phrase order). We emphasize techniques which made it practical to apply inside-outside estimation of a lexicalized context free grammar to such a language. These techniques relate to the treatment of argument cancellation and scrambled phrase order; to the treatment of case features in category labels; to the category vocabulary for nouns, articles, adjectives and their projections; to lexicalization based on uninflected lemmata rather than word forms; and to exploitation of a parameter-tying feature.

\section{Corpus and morphology}

The data for the experiment is a corpus of German subordinate clauses extracted by regular expression matching from a 200 million token newspaper corpus. The clause length ranges between four and 12 words. Apart from infinitival VPs as verbal arguments, there are no further clausal embeddings, and the clauses do not contain any punctuation except for a terminal period. The corpus contains 4128873 tokens and 450526 clauses which yields an average of 9.16456 tokens per clause. Tokens are automatically annotated with a list of part-of-speech (PoS) tags using a computational morphological analyser based on finite-state technology (Karttunen et al. (1994), Schiller and Stöckert (1995)).

A problem for practical inside-outside estimation of an inflectional language like German arises with the large number of terminal and low-level non-terminal categories in the grammar resulting from the morpho-syntactic features of words. Apart from major class (noun, adjective, and so forth) the analyser provides an ambiguous word with a list of possible combinations of inflectional features like gender, person, number (cf. the top part of Fig. 1 for an example ambiguous between nominal and adjectival PoS; the PoS is indicated following the ' + ' sign). In order to reduce the number of parameters to be estimated, and to reduce the size of the parse forest used in inside-outside estimation, we collapsed the inflectional readings of adjectives, adjective derived nouns, article words, and pronouns to a single morphological feature (see of Fig. 1 for an example). This reduced the number of low-level categories, as exemplified in Fig. 2: das has one reading as an article and one as a demonstrative; westdeutschen has one reading as an adjective, with its morphological feature $\mathrm{N}$ indicating the inflectional suffix.

We use the special tag UNTAGGED indicating that the analyser fails to provide a tag for the word. The vast majority of UNTAGGED words are proper names not recognized as such. These gaps in the morphology have little effect on our experiment.

\section{Grammar}

The grammar is a manually developed headed context-free phrase structure grammar for German subordinate clauses with 5508 rules and 


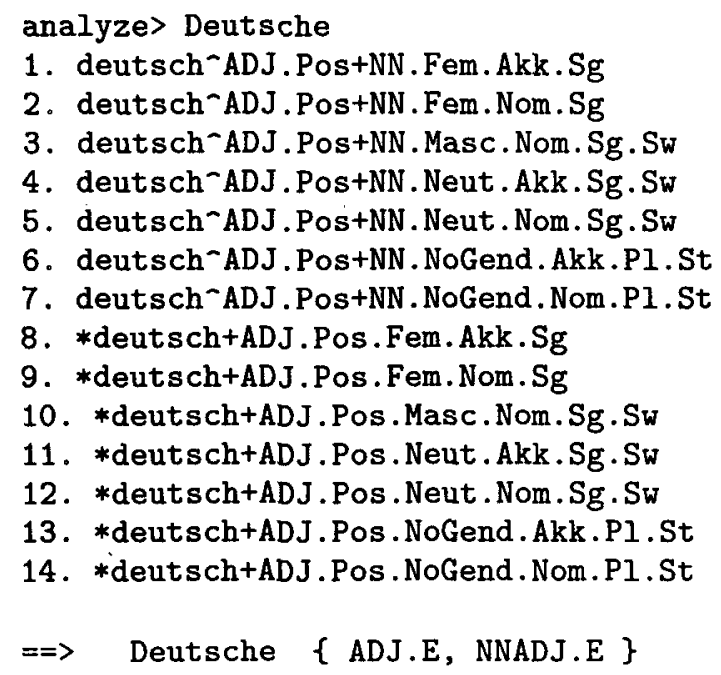

Figure 1: Collapsing Inflectional Features

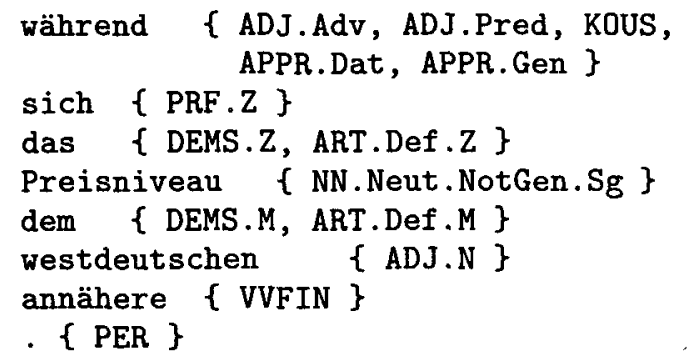

Figure 2: Corpus Clip

562 categories, 209 of which are terminal categories. The formalism is that of Carroll and Rooth (1998), henceforth C+R:

mother $\rightarrow$ non-heads head' non-heads (freq)

The rules are head marked with a prime. The non-head sequences may be empty. freq is a rule frequency, which is initialized randomly and subsequently estimated by the inside outsidealgorithm. To handle systematic patterns related to features, rules were generated by Lisp functions, rather than being written directly in the above form. With very few exceptions (rules for coordination, S-rule), the rules do not have more than two daughters.

Grammar development is facilitated by a chart browser that permits a quick and efficient discovery of grammar bugs (Carroll, 1997a). Fig. 3 shows that the ambiguity in the chart is quite considerable even though grammar and corpus are restricted. For the entire corpus, we computed an average 9202 trees per clause. In the chart browser, the categories filling the cells indicate the most probable category for that span with their estimated frequencies. The pop-up window under IP presents the ranked list of all possible categories for the covered span. Rules (chart edges) with frequencies can be viewed with a further menu. In the chart browser, colors are used to display frequencies (between 0 and 1 ) estimated by the inside-outside algorithm. This allows properties shared across tree analyses to be checked at a glance; often grammar and estimation bugs can be detected without mouse operations.

The grammar covers $88.5 \%$ of the clauses and $87.9 \%$ of the tokens contained in the corpus. Parsing failures are mainly due to UNTAGGED words contained in $6.6 \%$ of the failed clauses, the pollution of the corpus by infinitival constructions $(\approx 1.3 \%)$, and a number of coordinations not covered by the grammar $(\approx 1.6 \%)$.

\subsection{Case features and agreement}

On nominal categories, in addition to the four cases Nom, Gen, Dat, and Akk, case features with a disjunctive interpretation (such as Dir for Nom or Akk) are used. The grammar is written in such a way that non-disjunctive features are introduced high up in the tree. This results in some reduction in the size of the parse forest, and some parameter pooling. Essentially the full range of agreement inside the noun phrase is enforced. Agreement between the nominative NP and the tensed verb (e.g. in number) is not enforced by the grammar, in order to control the number of parameters and rules.

For noun phrases we employ Abney's chunk grammar organization (Abney, 1996). The noun chunk (NC) is an approximately non-recursive projection that excludes post-head complements and (adverbial) adjuncts introduced higher than pre-head modifiers and determiners but includes participial pre-modifiers with their complements. Since we perform complete context free parsing, parse forest construction, and inside-outside estimation, chunks are not motivated by deterministic parsing. Rather, they facilitate evaluation and graphical debugging, by tending to increase the span of constituents with high estimated frequency. 


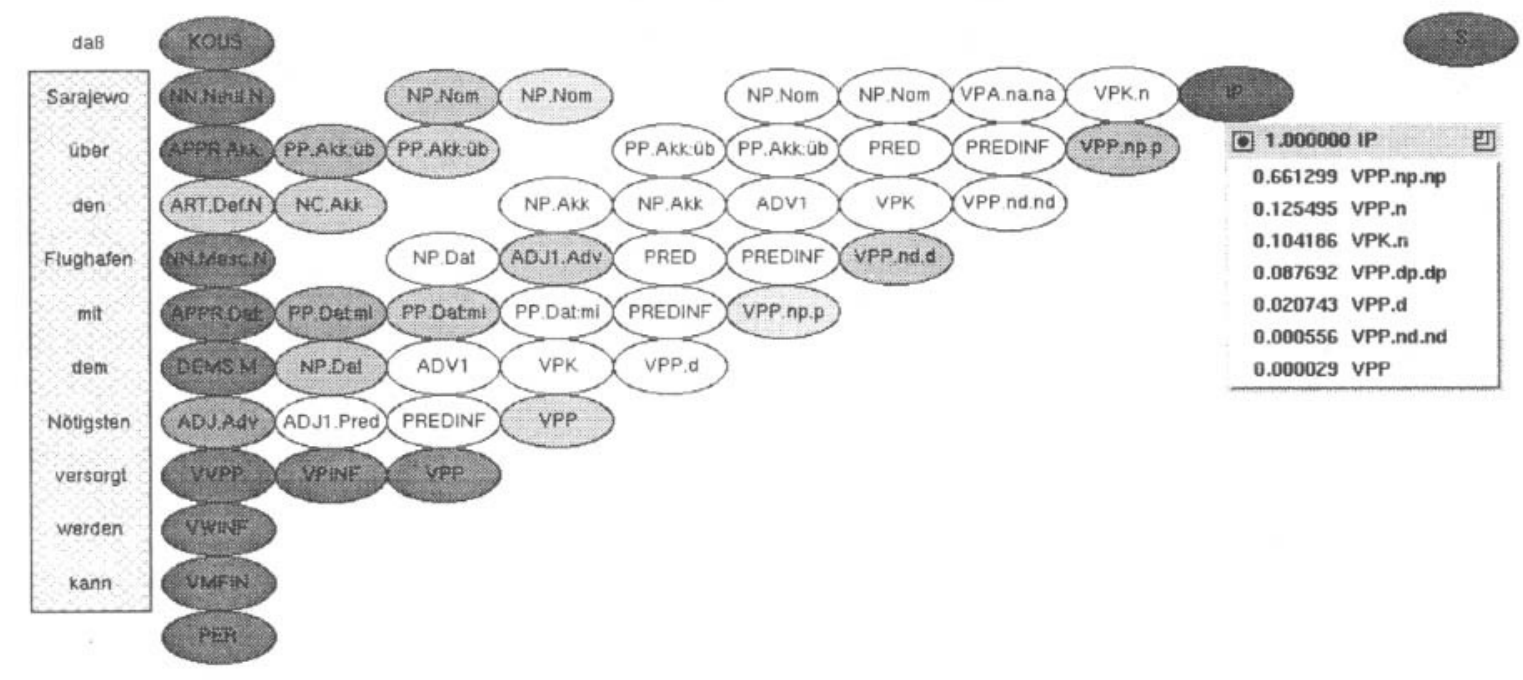

Figure 3: Chart browser

Word-by-word gloss of the clause: 'that Sarajevo over the airport with the essentials supplied will can'

\begin{tabular}{|c|c|c|}
\hline class & \# & frame types \\
\hline VPA & 15 & $\begin{array}{l}\text { n, na, nad, nai, nap, nar, nd, ndi, } \\
\text { ndp, ndr, ni, nir, np, npr, nr }\end{array}$ \\
\hline VPP & 13 & $\begin{array}{l}\mathrm{d}, \mathrm{di}, \mathrm{dp}, \mathrm{dr}, \mathrm{i}, \mathrm{ir}, \mathrm{n}, \mathrm{nd}, \mathrm{ni}, \mathrm{np}, \mathrm{p} \text {, } \\
\mathrm{pr}, \mathrm{r}\end{array}$ \\
\hline VPI & 10 & $\mathrm{a}, \mathrm{ad}, \mathrm{ap}, \mathrm{ar}, \mathrm{d}, \mathrm{dp}, \mathrm{dr}, \mathrm{p}, \mathrm{pr}, \mathrm{r}$ \\
\hline VPK & 2 & $\mathrm{i}, \mathrm{n}$ \\
\hline
\end{tabular}

Figure 4: Number and types of verb frames

\subsection{Subcategorisation frames of verbs}

The grammar distinguishes four subcategorisation frame classes: active (VPA), passive (VPP), infinitival (VPI) frames, and copula constructions (VPK). A frame may have maximally three arguments. Possible arguments in the frames are nominative (n), dative (d) and accusative (a) NPs, reflexive pronouns ( $r$ ), PPs (p), and infinitival VPs (i). The grammar does not distinguish plain infinitival VPs from $z u$-infinitival VPs. The grammar is designed to partially distinguish different $\mathrm{PP}$ frames relative to the prepositional head of the PP. A distinct category for the specific preposition becomes visible only when a subcategorized preposition is cancelled from the subcat list. This means that specific prepositions do not figure in the evaluation discussed below. The number and the types of frames in the different frame classes are given in figure 4.

German, being a language with comparatively free phrase order, allows for scrambling of arguments. Scrambling is reflected in the particu-
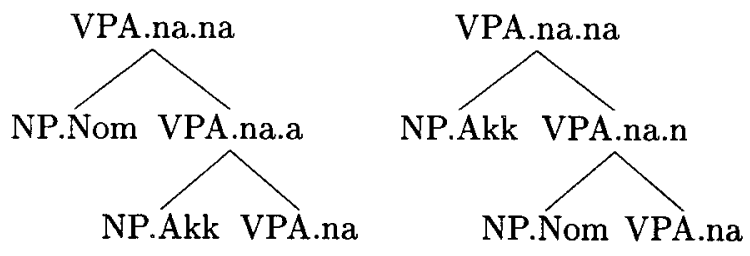

Figure 5: Coding of canonical and scrambled argument order

lar sequence in which the arguments of the verb frame are saturated. Compare figure 5 for an example of a canonical subject-object order in an active transitive frame and its scrambled objectsubject order. The possibility of scrambling verb arguments yields a substantial increase in the number of rules in the grammar (e.g. $102 \mathrm{com}$ binatorically possible argument rules for all in VPA frames). Adverbs and non-subcategorized PPs are introduced as adjuncts to VP categories which do not saturate positions in the subcat frame.

In earlier experiments, we employed a flat clausal structure, with rules for all permutations of complements. As the number of frames increased, this produced prohibitively many rules, particularly with the inclusion of adjuncts.

\section{Parameters}

The parameterization is as in $\mathrm{C}+\mathrm{R}$, with one significant modification. Parameters consist of (i) rule parameters, corresponding to right hand 
sides conditioned by parent category and parent head; (ii) lexical choice parameters for nonhead children, corresponding to child lemma conditioned by child category, parent category, and parent head lemma. See $\mathrm{C}+\mathrm{R}$ or Charniak (1995) for an explanation of how such parameters define a probabilistic weighting of trees. The change relative to $\mathrm{C}+\mathrm{R}$ is that lexicalization is by uninflected lemma rather than word form. This reduces the number of lexical parameters, giving more acceptable model sizes and eliminating splitting of estimated frequencies among inflectional forms. Inflected forms are generated at the leaves of the tree, conditioned on terminal category and lemma. This results in a third family of parameters, though usually the choice of inflected form is deterministic.

A parameter pooling feature is used for argument filling where all parent categories of the form VP.x.y are mapped to a category VP.x in defining lexical choice parameters. The consequence is e.g. that an accusative daughter of a nominative-accusative verb uses the same lexical choice parameter, whether a default or scrambled word order is used. (This feature was used by $\mathrm{C}+\mathrm{R}$ for their phrase trigram grammar, not in the linguistic part of their grammar.) Not all desirable parameter pooling can be expressed in this way, though; for instance rule parameters are not pooled, and so get split when the parent category bears an inflectional feature.

\section{Estimation}

The training of our probabilistic CFG proceeds in three steps: (i) unlexicalized training with the supar parser, (ii) bootstrapping a lexicalized model from the trained unlexicalized one with the ultra parser, and finally (iii) lexicalized training with the hypar parser (Carroll, 1997b). Each of the three parsers uses the insideoutside algorithm. supar and ultra use an unlexicalized weighting of trees, while hypar uses a lexicalized weighting of trees. ultra and hypar both collect frequencies for lexicalized rule and lexical choice events, while supar collects only unlexicalized rule frequencies.

Our experiments have shown that training an unlexicalized model first is worth the effort. Despite our use of a manually developed grammar that does not have to be pruned of superfluous rules like an automatically generated grammar,

\begin{tabular}{|c|c|c|c|c|c|}
\hline \multicolumn{2}{|r|}{ A } & \multicolumn{2}{|r|}{$\mathbf{B}$} & \multicolumn{2}{|r|}{ C } \\
\hline 1: & 52.0199 & 1: & 53.7654 & 1: & 49.8165 \\
\hline 2: & 25.3652 & 2: & 26.3184 & 2: & 23.1008 \\
\hline 3: & 24.5905 & 3: & 25.5035 & 3: & 22.4479 \\
\hline : & $\vdots$ & $\vdots$ & : & $\vdots$ & : \\
\hline 13: & 24.2872 & 55: & 25.0548 & 70: & 22.1445 \\
\hline 14: & 24.2863 & 56: & 25.0549 & 80: & 22.1443 \\
\hline 15: & 24.2861 & 57: & 25.0549 & 90: & 22.1443 \\
\hline 16: & 24.2861 & 58: & 25.0549 & 95: & 22.1443 \\
\hline 17: & 24.2867 & 59: & 25.055 & 96: & 22.1444 \\
\hline
\end{tabular}

Figure 6: Overtraining (iteration: cross-entropy on heldout data)

the lexicalized model is notably better when preceded by unlexicalized training (see also Ersan and Charniak (1995) for related observations). A comparison of immediate lexicalized training (without prior training of an unlexicalized model) and our standard training regime that involves preliminary unlexicalized training speaks in favor of our strategy (cf. the different 'lex 0 ' and 'lex 2' curves in figures 8 and 9). However, the amount of unlexicalized training has to be controlled in some way.

A standard criterion to measure overtraining is to compare log-likelihood values on held-out data of subsequent iterations. While the loglikelihood value of the training data is theoretically guaranteed to converge through subsequent iterations, a decreasing log-likelihood value of the held-out data indicates overtraining. Instead of log-likelihood, we use the inversely proportional cross-entropy measure. Fig. 6 shows comparisons of different sizes of training and heldout data (training/heldout): (A) $50 \mathrm{k} / 50 \mathrm{k}$, (B) $500 \mathrm{k} / 500 \mathrm{k}$, (C) $4.1 \mathrm{M} / 500 \mathrm{k}$. The overtraining effect is indicated by the increase in cross-entropy from the penultimate to the ultimate iteration in the tables. Overtraining results for lexicalized models are not yet available.

However, a comparison of precision/recall measures on categories of different complexity through iterative unlexicalized training shows that the mathematical criterion for overtraining may lead to bad results from a linguistic point of view. While we observed more or less converging precision/recall measures for lower level structures such as noun chunks, iterative unlexicalized training up to the overtraining threshold turned out to be disastrous for the evaluation of complex categories that depend on almost the 


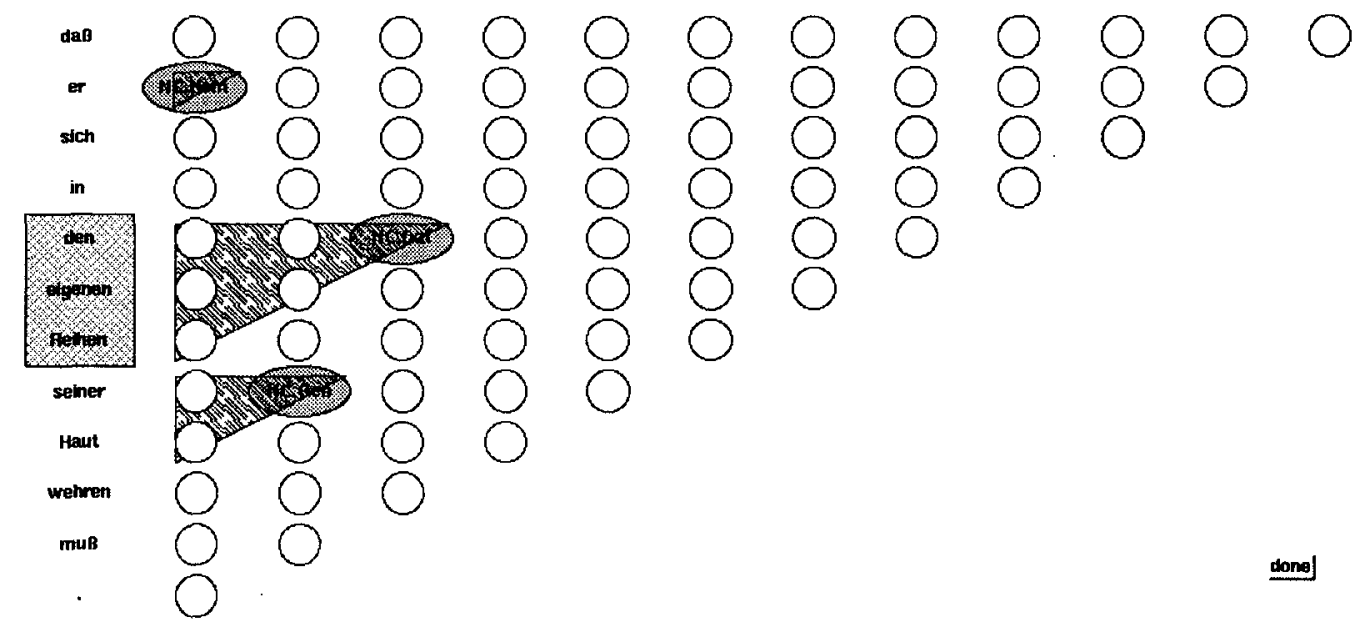

Figure 7: Chart browser for manual NC labelling

entire span of the clause. The recognition of subcategorization frames through 60 iterations of unlexicalized training shows a massive decrease in precision/recall from the best to the last iteration, even dropping below the results with the randomly initialized grammar (see Fig. 9).

\subsection{Training regime}

We compared lexicalized training with respect to different starting points: a random unlexicalized model, the trained unlexicalized model with the best precision/recall results, and an unlexicalized model that comes close to the crossentropy overtraining threshold. The details of the training steps are as follows:

(1) 0,2 and 60 iterations of unlexicalized parsing with supar;

(2) lexicalization with ultra using the entire corpus;

(3) 23 iterations of lexicalized parsing with hypar.

The training was done on four machines (two $167 \mathrm{MHz}$ UltraSPARC and two $296 \mathrm{MHz}$ SUNW UltraSPARC-II). Using the grammar described here, one iteration of supar on the entire corpus takes about 2.5 hours, lexicalization and generating an initial lexicalized model takes more than six hours, and an iteration of lexicalized parsing can be done in 5.5 hours.

\section{Evaluation}

For the evaluation, a total of 600 randomly selected clauses were manually annotated by two labellers. Using a chart browser, the labellers filled the appropriate cells with category names of NCs and those of maximal VP projections (cf. Figure 7 for an example of NC-labelling). Subsequent alignment of the labellers decisions resulted in a total of 1353 labelled NC categories (with four different cases). The total of 584 labelled VP categories subdivides into 21 different verb frames with 340 different lemma heads. The dominant frames are active transitive (164 occurrences) and active intransitive (117 occurrences). They represent almost half of the annotated frames. Thirteen frames occur less than ten times, five of which just once.

\subsection{Methodology}

To evaluate iterative training, we extracted maximum probability (Viterbi) trees for the 600 clause test set in each iteration of parsing. For extraction of a maximal probability parse in unlexicalized training, we used Schmid's lopar parser (Schmid, 1999). Trees were mapped to a database of parser generated markup guesses, and we measured precision and recall against the manually annotated category names and spans. Precision gives the ratio of correct guesses over all guesses, and recall the ratio of correct guesses over the number of phrases identified by human annotators. Here, we render only the precision/recall results on pairs of category names and spans, neglecting less interesting measures on spans alone. For the figures of adjusted recall, the number of unparsed misses has been subtracted from the number of possibilities. 


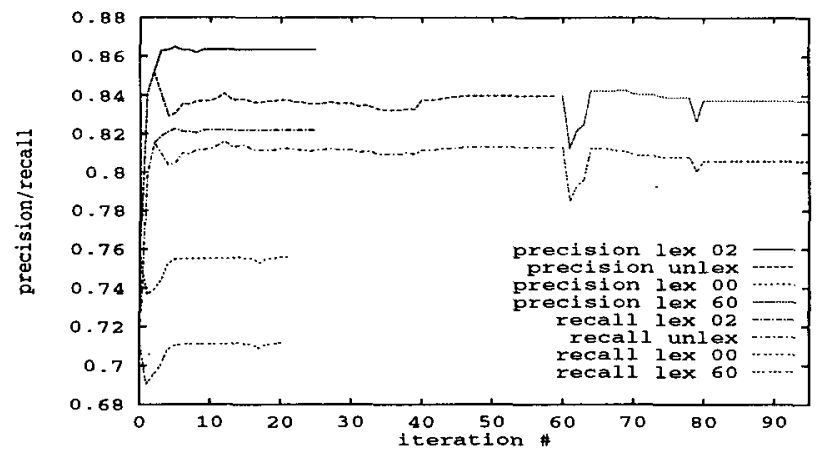

Figure 8: Precision/recall measures on NC cases

In the following, we focus on the combination of the best unlexicalized model and the lexicalized model that is grounded on the former.

\subsection{NC Evaluation}

Figure 8 plots precision/recall for the training runs described in section 5.1, with lexicalized parsing starting after 0,2 , or 60 unlexicalized iterations. The best results are achieved by starting with lexicalized training after two iterations of unlexicalized training. Of a total of 1353 annotated NCs with case, 1103 are correctly recognized in the best unlexicalized model and 1112 in the last lexicalized model. With a number of 1295 guesses in the unlexicalized and 1288 guesses in the final lexicalized model, we gain $1.2 \%$ in precision $(85.1 \%$ vs. $86.3 \%$ ) and $0.6 \%$ in recall $(81.5 \%$ vs. $82.1 \%)$ through lexicalized training. Adjustment to parsed clauses yields $88 \%$ vs. $89.2 \%$ in recall. As shown in Figure 8, the gain is achieved already within the first iteration; it is equally distributed between corrections of category boundaries and labels.

The comparatively small gain with lexicalized training could be viewed as evidence that the chunking task is too simple for lexical information to make a difference. However, we find about $7 \%$ revised guesses from the unlexicalized to the first lexicalized model. Currently, we do not have a clear picture of the newly introduced errors.

The plots labeled " 00 " are results for lexicalized training starting from a random initial grammar. The precision measure of the first lexicalized model falls below that of the unlexicalized random model ( $74 \%$ ), only recovering through lexicalized training to equalize the precision measure of the random model $(75.6 \%)$.

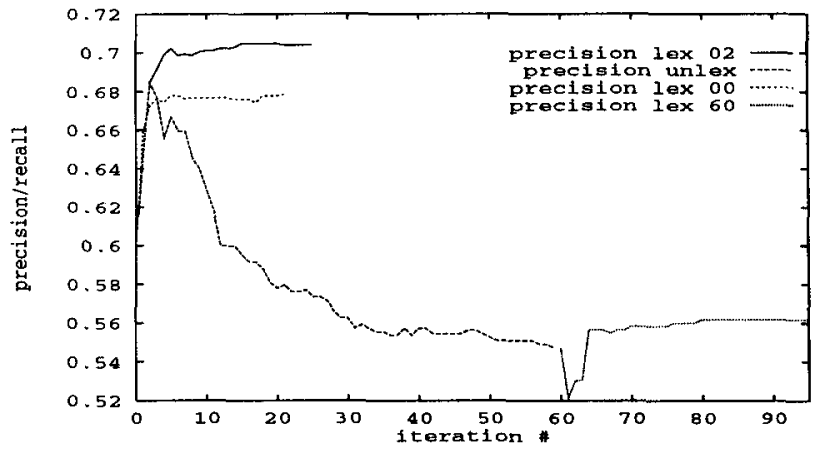

Figure 9: Precision measures on all verb frames

This indicates that some degree of unlexicalized initialization is necessary, if a good lexicalized model is to be obtained.

(Skut and Brants, 1998) report $84.4 \%$ recall and $84.2 \%$ for NP and PP chunking without case labels. While these are numbers for a simpler problem and are slightly below ours, they are figures for an experiment on unrestricted sentences. A genuine comparison has to await extension of our model to free text.

\subsection{Verb Frame Evaluation}

Figure 9 gives results for verb frame recognition under the same training conditions. Again, we achieve best results by lexicalising the second unlexicalized model. Of a total of 584 annotated verb frames, 384 are correctly recognized in the best unlexicalized model and 397 through subsequent lexicalized training. Precision for the best unlexicalized model is $68.4 \%$. This is raised by $2 \%$ to $70.4 \%$ through lexicalized training; recall is $65.7 \% / 68 \%$; adjustment by 41 unparsed misses makes for $70.4 \% / 72.8 \%$ in recall. The rather small improvements are in contrast to 88 differences in parser markup, i.e. $15.7 \%$, between the unlexicalized and second lexicalized model. The main gain is observed within the first two iterations (cf. Figure 9; for readability, we dropped the recall curves when more or less parallel to the precision curves).

Results for lexicalized training without prior unlexicalized training are better than in the $\mathrm{NC}$ evaluation, but fall short of our best results by more than $2 \%$.

The most notable observation in verb frame evaluation is the decrease of precision of frame recognition in unlexicalized training from the second iteration onward. After several dozen it- 


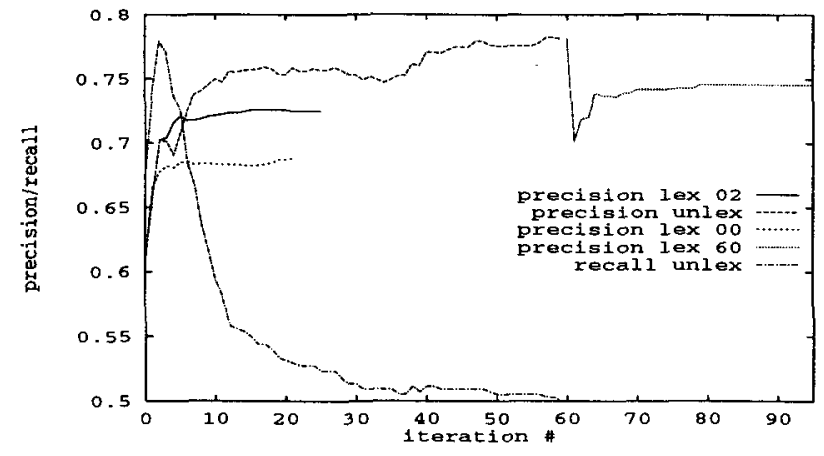

Figure 10: Precision measures on non-PP frames

erations, results are $5 \%$ below a random model and $14 \%$ below the best model. The primary reason for the decrease is the mistaken revision of adjoined PPs to argument PPs. E.g. the required number of 164 transitive frames is missed by 76 , while the parser guesses 64 VPA.nap frames in the final iteration against the annotator's baseline of 12 . In contrast, lexicalized training generally stabilizes w.r.t. frame recognition results after only few iterations.

The plot labeled "lex 60 " gives precision for a lexicalized training starting from the unlexicalized model obtained with 60 iterations, which measured by linguistic criteria is a very poor state. As far as we know, lexicalized EM estimation never recovers from this bad state.

\subsection{Evaluation of non-PP Frames}

Because examination of individual cases showed that PP attachments are responsible for many errors, we did a separate evaluation of non-PP frames. We filtered out all frames labelled with a PP argument from both the maximal probability parses and the manually annotated frames (91 filtered frames), measuring precision and recall against the remaining 493 labeller annotated non-PP frames.

For the best lexicalized model, we find somewhat but not excessively better results than those of the evaluation of the entire set of frames. Of 527 guessed frames in parser markup, 382 are correct, i.e. a precision of $72.5 \%$. The recall figure of $77.5 \%$ is considerably better since overgeneration of 34 guesses is neglected. The differences with respect to different starting points for lexicalization emulate those in the evaluation of all frames.

The rather spectacular looking precision and recall differences in unlexicalized training con- firm what was observed for the full frame set. From the first trained unlexicalized model throughout unlexicalized training, we find a steady increase in precision ( $70 \%$ first trained model to $78 \%$ final model) against a sharp drop in recall $(78 \%$ peek in the second model vs. $50 \%$ in the final). Considering our above remarks on the difficulties of frame recognition in unlexicalized training, the sharp drop in recall is to be expected: Since recall measures the correct parser guesses against the annotator's baseline, the tendency to favor PP arguments over PP-adjuncts leads to a loss in guesses when PP-frames are abandoned. Similarly, the rise in precision is mainly explained by the decreasing number of guesses when cutting out non-PP frames. For further discussion of what happens with individual frames, we refer the reader to (Beil et al., 1998).

One systematic result in these plots is that performance of lexicalized training stabilizes after a few iterations. This is consistent with what happens with rule parameters for individual verbs, which are close to their final values within five iterations.

\section{Conclusion}

Our principal result is that scrambling-style free-er phrase order, case morphology and subcategorization, and NP-internal gender, number and case agreement can be dealt with in a head-lexicalized PFCG formalism by means of carefully designed categories and rules which limit the size of the packed parse forest and give desirable pooling of parameters. Hedging this, we point out that we made compromises in the grammar (notably, in not enforcing nominativeverb agreement) in order to control the number of categories, rules, and parameters.

A second result is that iterative lexicalized inside-outside estimation appears to be beneficial, although the precision/recall increments are small. We believe this is the first substantial investigation of the utility of iterative lexicalized inside-outside estimation of a lexicalized probabilistic grammar involving a carefully built grammar where parses can be evaluated by linguistic criteria.

A third result is that using too many unlexicalized iterations (more than two) is detrimental. A criterion using cross-entropy overtraining 
on held-out data dictates many more unlexicalized iterations, and this criterion is therefore inappropriate.

Finally, we have clear cases of lexicalized EM estimation being stuck in linguistically bad states. As far as we know, the model which gave the best results could also be stuck in a comparatively bad state. We plan to experiment with other lexicalized training regimes, such as ones which alternate between different training corpora.

The experiments are made possible by improvements in parser and hardware speeds, the carefully built grammar, and evaluation tools. In combination, these provide a unique environment for investigating training regimes for lexicalized PCFGs. Much work remains to be done in this area, and we feel that we are just beginning to develop understanding of the time course of parameter estimation, and of the general efficacy of EM estimation of lexicalized PCFGs as evaluated by linguistic criteria.

We believe our current grammar of German could be extended to a robust free-text chunk/phrase grammar in the style of the English grammar of Carroll and Rooth (1998) with about a month's work, and to a free-text grammar treating verb-second clauses and additional complementation structures (notably extraposed clausal complements) with about one year of additional grammar development and experiment. These increments in the grammar could easily double the number of rules. However this would probably not pose a problem for the parsing and estimation software.

\section{References}

Steven Abney. 1996. Chunk stylebook. Technical report, SfS, Universität Tübingen.

Franz Beil, Glenn Carroll, Detlef Prescher, Stefan Riezler, and Mats Rooth. 1998. Insideoutside estimation of a lexicalized PCFG for German. -Gold-. In Inducing Lexicons with the EM Algorithm. AIMS Report 4(3), IMS, Universität Stuttgart.

Glenn Carroll and Mats Rooth. 1998. Valence induction with a head-lexicalized PCFG. In Proceedings of EMNLP-3, Granada.

Glenn Carroll, 1997a. Manual pages for charge, hyparCharge, and tau. IMS, Universität Stuttgart.
Glenn Carroll, 1997b. Manual pages for supar, ultra, hypar, and genDists. IMS, Stuttgart University.

E. Charniak. 1995. Parsing with context-free grammars and word statistics. Technical Report CS-95-28, Department of Computer Science, Brown University.

M. Ersan and E. Charniak. 1995. A statistical syntactic disambiguation program and what it learns. Technical Report CS-95-29, Department of Computer Science, Brown University.

Lauri Karttunen, Todd Yampol, and Gregory Grefenstette, 1994. INFL Morphological Analyzer/Generator 3.2.9 (3.6.4). Xerox Corporation.

Anne Schiller and Chris Stöckert, 1995. DMOR. IMS, Universität Stuttgart.

Helmut Schmid, 1999. Manual page for lopar. IMS, Universität Stuttgart.

Wojciech Skut and Thorsten Brants. 1998. A maximum-entropy partial parser for unrestricted text. In Proceedings of the Sixth Workshop on Very Large Corpora, Montréal, Québec. 